\title{
Bacteriological Profile and Antimicrobial Susceptibility Patterns of Blood Borne Pathogens in a Tertiary Care Center, Jodhpur (Rajasthan), India
}

\author{
R.S. Parihar, Dallaram*, P.K. Khatri, Archana and Deep Shikhar Acharya \\ Department of Microbiology, Dr. S. N. Medical College, Jodhpur, Rajasthan, India \\ *Corresponding author
}

\begin{tabular}{|l|}
\hline Ke y w o r d s \\
BacT/alert, Blood stream \\
infection, Blood samples, \\
Bacterial isolate, \\
Septicemia, \\
Antimicrobial \\
susceptibility
\end{tabular}

Blood stream infections cause a significant morbidity and mortality worldwide. Rapid and reliable detection of bacterial pathogens and rational use of antimicrobial are required for proper management. The Aims of this study includes Isolation and identification of pathogens from blood samples using automated BacT/ALERT blood culture system and their antibiotic resistance patterns. This 6 months study covered 387 blood culture samples from septicemia patients in which 80 samples were positive for aerobic bacterial isolates. As per standard protocol further bacteriological identification and antimicrobial susceptibility testing were performed in which the most common organisms were CONS (33) and Klebsiella spp (07) in gram positive and gram negative organisms respectively. The gram negative organisms were multi drug resistance with a very high resistance to beta-lactam antibiotics except imipenem which is sensitive to all strains, whereas gram positive organisms are mostly resistant to penicillin followed by erythromycin, clindamycin, levofloxacin and are completely sensitive to vancomycin. The present study shows the bacteriological etiology of sepsis along with their antimicrobial susceptibility pattern of septicemic isolates that may provide necessary information for the formulation of antibiotic policy and prevent nosocomial infection for effective management of such cases.

\section{Introduction}

Worldwide, Blood stream infections (BSI) constitute a major cause of severe morbidity and mortality in hospitalized patients. Blood stream infections range from self-limiting infections to life threatening sepsis which requires rapid and appropriate antimicrobial treatment. In India and developing countries septicemia is an important cause of illness and death among hospitalized patients (Sharma et al., 1987; Diekma et al., 2003). For improving the antimicrobial treatment to patients of septicemia, monitoring of blood culture isolates and determination of susceptibility to antibiotics are necessary (Chitralekha Saikumar et al., 2015). Many bacterial pathogens have developed resistance to most of the antibiotics; economically and socially, it has become a serious health problem all over the worlds (Jo Ann, 2009), there are lots of microorganisms have been reported which cause septicemia with variation in distribution from place to place (Gohel et al., 2014). Rapid identification and antimicrobial susceptibility testing of the causative agents of bloodstream 
infections has to be done by the clinical microbiology laboratory so it provides essential information to clinicians for selecting appropriate antimicrobial therapy for patients with bloodstream infections (Lupetti et al., 2009). Automated blood culture system is continuous monitoring technology that reduces the time needed to detect positive blood cultures as well as decreases the specimen handling (Kim and Han, 2010).

The inoculated blood culture bottles should be transported to laboratory as early as possible and also the interval between the collection of blood and the entry of the bottles into an automated blood culture system should not be longer than 2 to 4 hours. Because of the changing epidemiology and susceptibility patterns of microorganisms emphasize the necessity of constant surveillance of blood stream infections, the present study was done to analyze various organisms causing bacteremia and their antibiotic resistance pattern. This study wide enable using appropriate antibiotic, may decrease the hospital stay and cost of treatment and reduce mortality.

The aims of this study include, isolation and identification of pathogens from blood samples using automated BacT/ALERT blood culture system and their antibiotic resistance patterns in a tertiary care center, Jodhpur (Rajasthan), India.

\section{Materials and Methods}

This study was carried out at the Department of Microbiology, Dr. S. N. Medical College, Jodhpur (Rajasthan), India. A total of 387 blood samples from clinically suspected cases of septicemia were studied during a 6 months period from Feb 2017 to July 2017. Blood samples were collected by using strict aseptic precautions and inoculated immediately into BacT/ALERT FA plus and PF plus aerobic blood culture bottles. After collection these bottles were immediately incubated in BacT/ALERT 3D (bioMerieoux) - a fully automated blood culture system for detection of growth in blood culture. The negative results were followed up to5-7days and final report was issued. The BacT/ALERT automatically gives a signal alert. The positive bottles were then subculture on blood, MacConkey and chocolate agar. These plates were incubated aerobically at $37^{\circ} \mathrm{C}$ and examined after 18-24 hours. Final identification was done by colony characteristics, Gram's staining, motility testing (hanging drop preparation) and routine biochemical test (Catalase, coagulase, indole, methyl red, citrate, urease, Triple sugar iron, PPA, and oxidase testing). Fungal isolate was identified by Gram's staining showing gram positive budding yeast cells and germ tube testing. Antimicrobial susceptibility testing of bacterial isolates was done by the Kirby-Bauer disc diffusion method using Muller Hinton agar media as per CLSI guidelines (Clinical and Laboratory Standards Institute, 2017).

Blood stream infections can lead to life threatening sepsis and require immediate antimicrobial treatment. Blood culture is an essential tool for the investigation of clinically suspected sepsis. The present study has been conducted to describe the profile of bacterial isolates from blood cultures and their antibiotic resistance. This is prospective study of 387 blood cultures, collected from clinically suspected cases of bacteremia studied over a period of six months in a tertiary care hospital in Jodhpur, Rajasthan. The isolates were identified by standard biochemical tests and antimicrobial resistance patterns were determined by CLSI guidelines. Blood cultures were positive in $80(20.7 \%)$ patients by BacT alert system. Gram positive organism accounted for $67.5 \%$ cases; most common being Coagulase negative Staphyococcus spp (41.3\%), Staphylococcus 
aureus (23.8\%) followed by Enterococci $(2.5 \%)$, Of the Gram negative isolates, Klebsiella spp $(8.8 \%)$ was the most common followed by Escherichia coli (5.0\%). Candida albicans was isolated in few cases. Gram positive isolates showed high resistance to penicillin $(75.9 \%)$ and least resistance to linezolid (1.9\%) and no resistance to vancomycin. Gram negative isolates were found high resistance to ampicillin (82.6\%) and ceftazidime $(73 \%)$ and no resistance to Imipenem. This study provides information on antibiotic resistance of blood isolates. It may be a useful guide for physicians initiating empiric therapy and will help in formulation of antibiotic therapy strategy.

\section{Results and Discussion}

During the study period, 387 blood culture samples were analyzed by automated blood culture system (Bact/Alert). Out of which 80 samples were culture positive and rest of 307 samples were culture negative. Amongst total positive samples 55 and 25 were male and female patients respectively. Among them majority of patients were from $0-1$ year age (23) followed by more than 50 years of age (18). Detail of age groups distribution was given in (Table 1 and Figure 1).

Out of 80 blood culture positive isolates, 54 were Gram positive organisms, 26 were Gram negatives organisms. Also 4 gram positive bacilli have been isolated, probably environmental contaminant and 1 candida species excluded from the positive isolates.

Detail description of isolates was shown in (Figure 2).

In this study two major isolates were Coagulase negative Staphylococci (33) and Staphylococcus aureus (19) followed by Klebsiella spp (07), Acinetobacter spp (06) and Escherichia coli (04).
Antibiotic susceptibility patterns of both gram positive organism and gram negative organisms are shown in Table 2 and 3 respectively. The susceptibility pattern of Staphylococcus aureus exhibit most resistance to penicillin (75.9\%) followed by erythromycin (63\%) and clindamycin (26.31\%). Another gram positive organism, Coagulase negative staphylococci, shows maximum resistance to erythromycin $(60.6 \%)$ followed by clindamycin (35.2\%) and levofloxacin $(27.8 \%)$. Among gram negative organisms Klebsiella spp was the predominant isolate which is mostly Resistance to ampicillin (76\%) and ceftazidime (73\%) followed by piperacillin/tazobactum (64\%), levofloxacin (58\%) and amikacin (50\%). In this study both gram positive organisms and gram negative organisms show $100 \%$ sensitivity to vancomycin and imipenem respectively (Fig. 3 and 4).

The findings which is obtained from this study showing that sepsis is one of the leading causes of death, and rapid identification of blood stream infection is mandatory to perform adequate antibiotic therapy.

In the present study a total of 387 blood culture samples were collected and analyzed, of which $80(20.7 \%)$ were positive by BacT/Alert system.

Which is quite similar to Sahoo et al., (2016) and Alam et al., (2011) but quite lower Kavitha et al., (2010) and Maimoona et al., (2014).

The incidence of Gram-positive organism is (67.5\%) while (32.5.\%) were Gram-negative organisms which was quite similar to Kalpesh et al., (2014) and China et al., (2013), but in other studies like Maimoona et al., (2014), and Ayobola et al., (2011) Gram-negative organisms have taken over Gram-positive organisms in hospital settings. 
Table.1 Age and gender wise distribution of blood culture of sepsis patients

\begin{tabular}{|c|c|c|c|}
\hline Age group & Male & Female & Total \\
\hline $0-1$ & 14 & 9 & 23 \\
\hline $1-10$ & 6 & 0 & 6 \\
\hline $\mathbf{1 1 - 2 0}$ & 5 & 6 & 11 \\
\hline $\mathbf{2 1 - 3 0}$ & 7 & 5 & 12 \\
\hline $\mathbf{3 1 - 4 0}$ & 7 & 0 & 7 \\
\hline $\mathbf{4 1 - 5 0}$ & 3 & 0 & 3 \\
\hline $\mathbf{5 0}$ & 13 & 5 & 18 \\
\hline
\end{tabular}

Table.2 Antibiotic Resistance pattern of gram positive bacteria

\begin{tabular}{|c|c|c|c|c|c|c|c|}
\hline $\begin{array}{l}\text { Organism } \\
\text { name }\end{array}$ & $\begin{array}{c}\text { Antibiotic } \\
\text { Susceptibility } \\
\text { test }\end{array}$ & 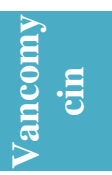 & $\begin{array}{l}\text { 을 } \\
\frac{8}{2} \\
.0\end{array}$ & 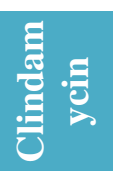 & $\sum_{\text {: }}^{2}$ & 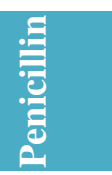 & 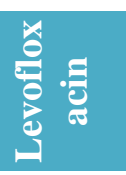 \\
\hline \multirow{2}{*}{$\begin{array}{c}\text { Staphylococcus } \\
\text { aureus } \\
\end{array}$} & Sensitive & 19 & 18 & 14 & 06 & 04 & 13 \\
\hline & Resistance & 00 & 01 & 05 & 13 & 15 & 06 \\
\hline \multirow[t]{2}{*}{ CoNS } & Sensitive & 33 & 33 & 20 & 13 & 08 & 25 \\
\hline & Resistance & 00 & 00 & 13 & 20 & 25 & 08 \\
\hline \multirow[t]{2}{*}{ Enterococcus } & Sensitive & 02 & 02 & 01 & 01 & 01 & 01 \\
\hline & Resistance & 00 & 00 & 01 & 01 & 01 & 01 \\
\hline \multirow[t]{2}{*}{ Total } & Sensitive & 54 & 53 & 35 & 20 & 13 & 39 \\
\hline & Resistance & 00 & 01 & 19 & 34 & 41 & 15 \\
\hline
\end{tabular}

Table.3 Antibiotic resistance pattern of gram negative bacteria
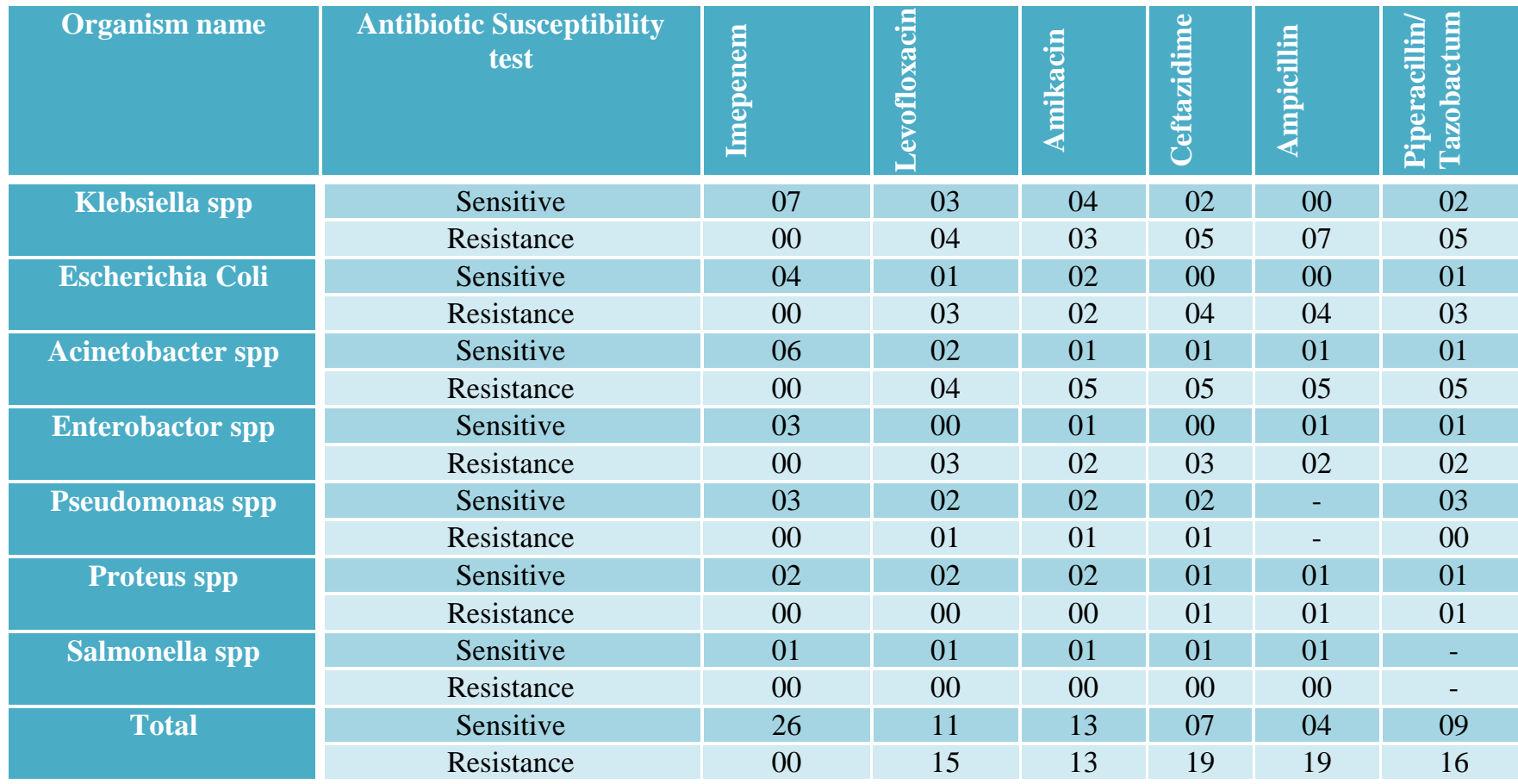

\begin{tabular}{|c|c|c|c|c|c|c|}
\hline Sensitive & 07 & 03 & 04 & 02 & 00 & 02 \\
\hline Resistance & 00 & 04 & 03 & 05 & 07 & 05 \\
\hline Sensitive & 04 & 01 & 02 & 00 & 00 & 01 \\
\hline Resistance & 00 & 03 & 02 & 04 & 04 & 03 \\
\hline Sensitive & 06 & 02 & 01 & 01 & 01 & 01 \\
\hline Resistance & 00 & 04 & 05 & 05 & 05 & 05 \\
\hline Sensitive & 03 & 00 & 01 & 00 & 01 & 01 \\
\hline Resistance & 00 & 03 & 02 & 03 & 02 & 02 \\
\hline Sensitive & 03 & 02 & 02 & 02 & - & 03 \\
\hline Resistance & 00 & 01 & 01 & 01 & - & 00 \\
\hline Sensitive & 02 & 02 & 02 & 01 & 01 & 01 \\
\hline Resistance & 00 & 00 & 00 & 01 & 01 & 01 \\
\hline Sensitive & 01 & 01 & 01 & 01 & 01 & - \\
\hline Resistance & 00 & 00 & 00 & 00 & 00 & - \\
\hline Sensitive & 26 & 11 & 13 & 07 & 04 & 09 \\
\hline Resistance & 00 & 15 & 13 & 19 & 19 & 16 \\
\hline
\end{tabular}


Fig.1 Age and gender wise distribution of blood culture of sepsis patients

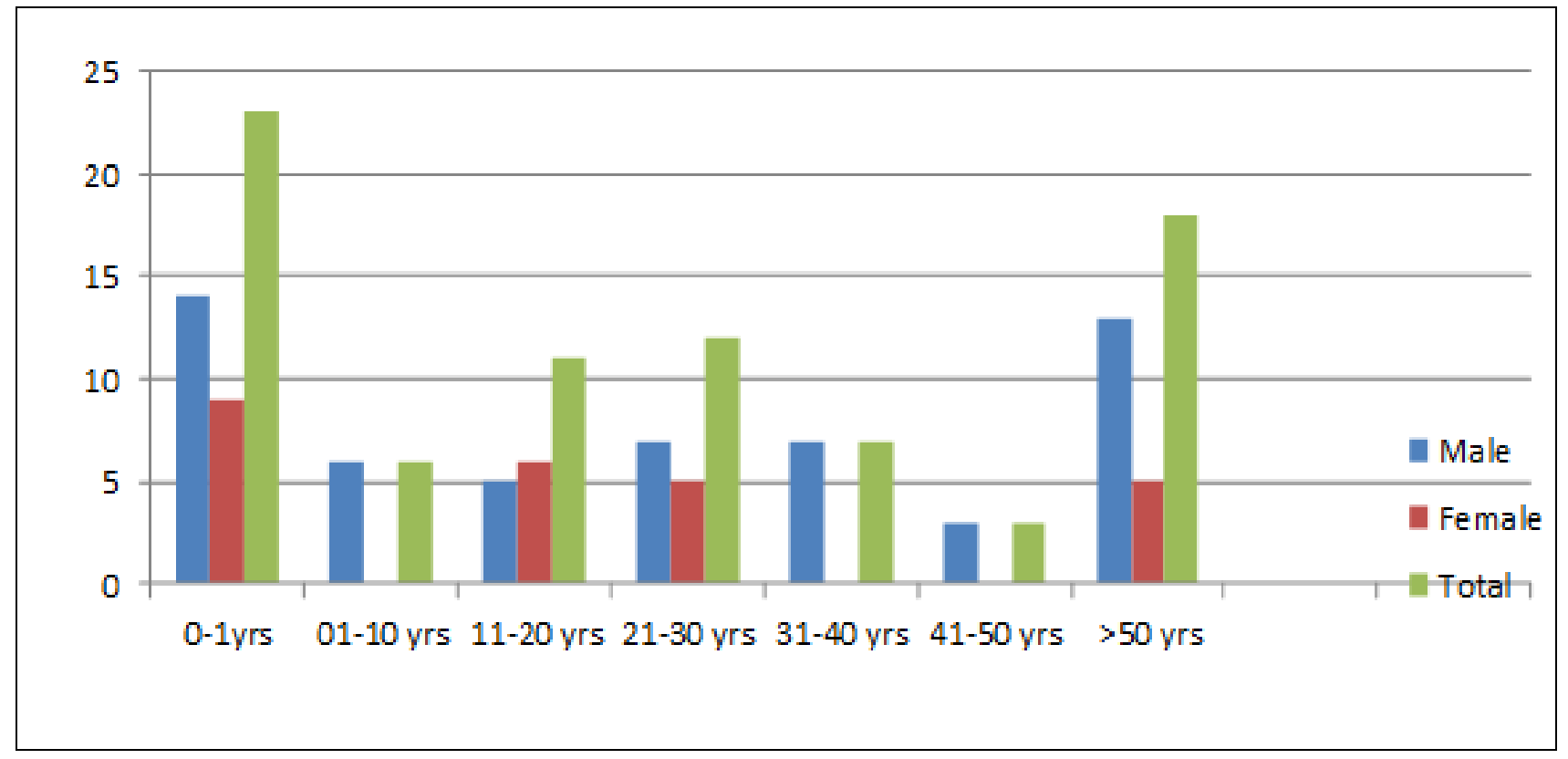

Fig.2 Organism isolated from positive blood samples

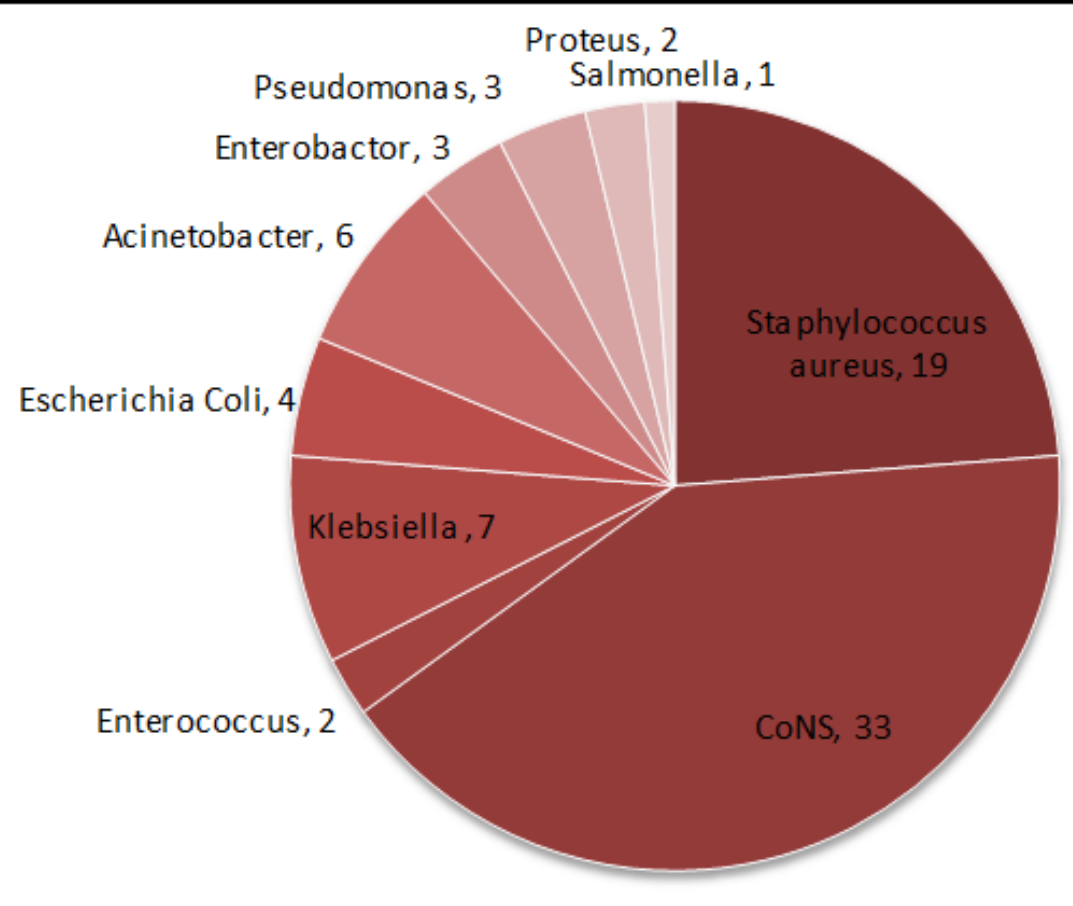


Fig.3 Antibiotic resistance pattern of gram positive bacteria

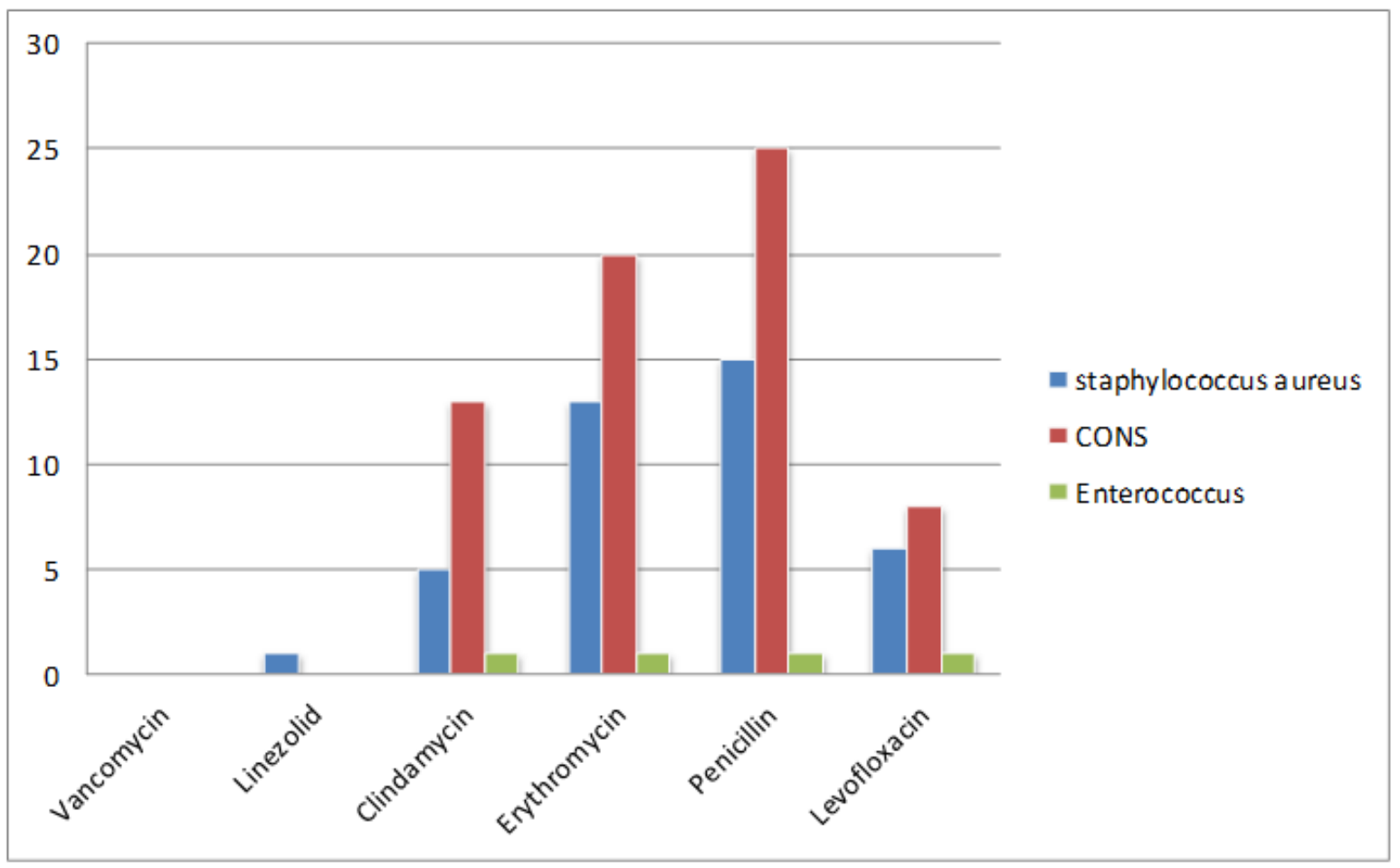

Fig.4 Antibiotic resistance pattern of gram negative bacteria

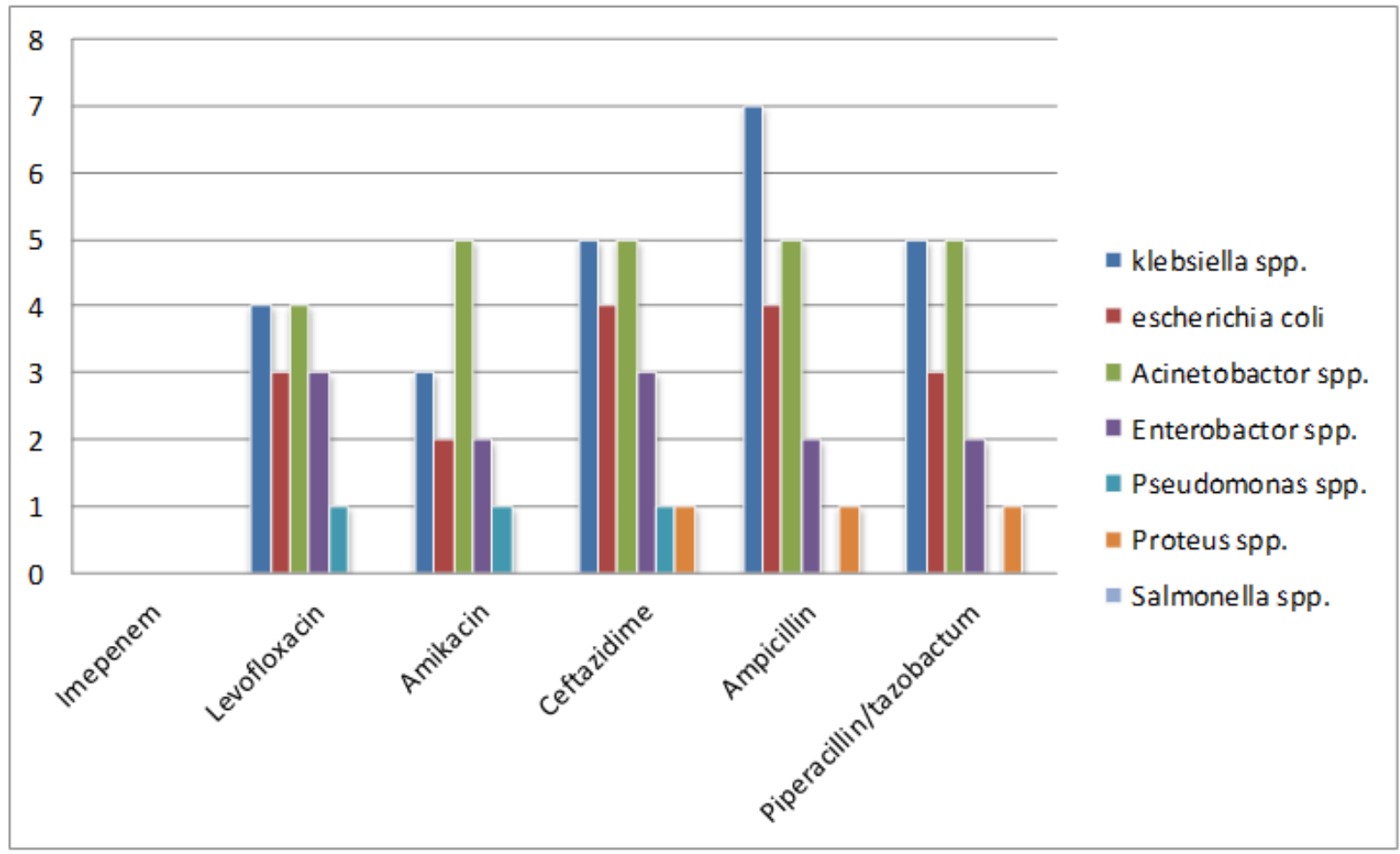


The present study indicates that infections by Gram-positive organisms constitute a significant threat to bacteremia and septicemia in our hospital setup.

This study shows that among the Gram positive organisms, Coagulase negative staphylococci is the commonest (41.25\%) isolate which is followed by Staphylococcus aureus (23.75\%) which is quite similar to study by Kalpesh et al., (2014) and Anbumani et al., (2008).

While as Klebsiella spp is the most predominant $(8.75 \%)$ isolate among gram negative organisms which is correlated with Panday et al., (2017).

In this study higher prevalence of antimicrobial resistance was noted, in gram negative organisms. This might be due to indiscriminate use of antibiotics in hospital.

Most of the gram negative organisms were multi drug resistance with a very high resistance to beta-lactam antibiotics except imipenem which is sensitive to all strains, whereas gram positive organisms are mostly resistant to penicillin followed by erythromycin, clindamycin, levofloxacin and are completely sensitive to vancomycin. These results are quite similar to Garg et al., (2007)

The rise in antibiotic resistance in blood isolates emphasizes the importance of sound hospital infection control, rational prescribing policies, and the need for awareness to use antimicrobial drugs.

It may be concluded from the study that early diagnosis and appropriate treatment of BSIs should be based on the current knowledge of bacterial profile and antibiotic resistance pattern, which should be provided by microbiology laboratory from time to time. We observed that Staphylococcus species and organisms belonging to Enterobacteriaceae family are the leading causes of septicemia. Increasing incidence of multidrug resistance organisms raises serious concerns and mandates strict antibiotic policy to prevent emergence and spread of antibiotic resistance. We hope that these results could support microbiologists, clinicians and hospital managers in the identification and implementation of strategic targeted actions to coordinate infection control interventions and antimicrobial policies in order to decrease the rate the emergence of resistance and minimize mortality of septicemia patients.

\section{References}

Alam, M.S., Pillai, P.K., Kapur, P., Pillai, K.K. 2011. Resistance patterns of bacteria isolated from bloodstream infections at a university hospital in Delhi. J. Pharm. Bioallied Sci., 3: 525-30.

Anbumani, N., et al., 2008. "Distribution and antimicrobial susceptibility of bacteria isolated from blood cultures of hospitalized patients in a tertiary care hospital, Indian J. Practicing Doctor, vol. 5, no. 2, pp. 1-7.

Ayobola, E.D., et al., 2011. "Study of prevalence and antimicrobial susceptibility of blood culture bacterial isolates," Malaysian J. Microbiol., vol. 7, no.2, pp.78-82.

China, D., and V. Gupta. 2013. "Bacteriological profile and antimicrobial susceptibility pattern of blood isolates from a tertiary care hospital in North India," Int. J. Pharmaceutical Res. Biosci., vol. 2, no. 2, pp. 24-35.

Chitralekha Saikumar, Bindu, Kiran Madhusudhan, Praveena, R., Illamani, V. 2015. Aerobic Microbial Profile and Antibiotic Susceptibility of Blood Isolates in a Tertiary Care Center, Int. J. Pharm. Sci. Rev. Res., 34(2), Article No. 23, Pages: 135-137.

Clinical and Laboratory Standards Institute. Performance standards for Antimicrobial susceptibility testing, $27^{\text {th }}$ international supplements. CLSI Document M100-S27. Wayne, Pennysylavania, USA: CLSI; 2017.

Debananda Sahoo, Lalatendu Mohanty, S.S. Panda, S.N., Mishra. 2016. Bacteriological Analysis of Blood Culture 
Isolates in Patients with Sepsis in A Tertiary Care Hospital of Eastern India, IJCMR, Volume 3 | Issue 12 | December 2016 | ICV (2015): 77.83.

Diekma DJ, Beekman SE, Chapin KC, Morel

KA, Munson E, Deorn GV: Epidemiology and outcome of nosocomial and community onset bloodstream infection. J Clin Microbiol 2003, 41:3655-3660.

Garg, A., et al., 2007. "Bacteriological profile and antimicrobial resistance of blood culture isolates from a university hospital," J. Indian Academy of Clin. Med., vol. 8, no. 2, pp. 139-143

Gohel, K., Jojera, A., Soni, S., Gang, S., Sabnis, R., Desai, M. 2014. Bacteriological profile and drug resistance patterns of blood culture isolates in a tertiary care nephrourology teaching institute. Biomed. Res. Int., 153747. doi: 10.1155/2014/ 153747.

Jo Ann, D. 2009. Antibiotic resistance: the ongoing challenge for effective drug therapy. JAAPA, 22(3): 18-22A.

Kalpesh Gohel, et al., 2014. Bacteriological profile and drug resistance patterns of blood culture isolates in a tertiary care nephrourology teaching institute, Hindawi Publishing Corporation BioMed Res. Int., Article ID 153747, 5 pages http://dx.doi.org/10.1155/2014/153747.

Kavitha, P., et al., 2010. "Bacteriological profile and antibiogram of blood culture isolates in a pediatric care unit," J. Laboratory Physicians, vol. 2, pp. 85-88.

Kim, K.E. and J.Y. Han, 2010. Evaluation of the clinical performance of an automated pro calcitonin assay for the quantitative detection of bloodstream infection. Korean. J. La. Med., 30: 153159. DOI:10.3343/kjlm.2010.30.2.153.

Lupetti, et al., 2009. Rapid identification and antimicrobial susceptibility testing of Gram-positive cocci in blood cultures by direct inoculation into the BD Phoenix system, Clin. Microbiol. Infect, Volume 16, Issue 7.

Maimoona Mustafa and Syed Laeeq Ahmed. 2014. Bacteriological profile and antibiotics susceptibility pattern in neonatal septicemia in view of emerging drug resistance, J. Med. Allied Sci., 4(1): 02-08 www. jmas.

Manjusha Pandey, Devendra Niranjan and R.C., Pande. 2017. Bacteriological Profile and Antimicrobial Resistance of Blood Culture Isolates from a 350 bedded Hospital Lucknow, India. Int. J. Curr. Microbiol. App. Sci., 6(1): 184193. doi: http://dx.doi.org/10.20546/ijcmas.2017. 601.023.

Sharma PP, Halder D, Dutta AK, Dutta R, Bhatnagar S, Bali A et al., Bacteriological profile of neonatal septicemia. Indian Pediatr 1987; 24:1011-7.

\section{How to cite this article:}

Parihar, R.S., Dallaram, P.K. Khatri, Archana and Deep Shikhar Acharya. 2018. Bacteriological Profile and Antimicrobial Susceptibility Patterns of Blood Borne Pathogens in a Tertiary Care Center, Jodhpur (Rajasthan), India. Int.J.Curr.Microbiol.App.Sci. 7(10): 1785-1792. doi: https://doi.org/10.20546/ijcmas.2018.710.203 